\title{
Sustained improvements in neurological function in spinal cord injured patients treated with oral 4-aminopyridine: three cases
}

\author{
PJ Potter ${ }^{1}$, KC Hayes ${ }^{1}$, JTC Hsieh ${ }^{1}$, GA Delaney ${ }^{1}$ and JL Segal ${ }^{2}$ \\ ${ }^{1}$ Department of Physical Medicine \& Rehabilitation, Parkwood Hospital and The University of Western Ontario, \\ London, Ontario, Canada; ${ }^{2}$ Department of Medicine, DVA Medical Center, Long Beach, California, USA
}

\begin{abstract}
Preclinical trials of intravenously administered 4-Aminopyridine (4-AP) have demonstrated transient improvements in neurological function in patients with longstanding spinal cord injury (SCI). The present report describes three patients with SCI who responded favourably in preclinical trials and who were subsequently administered oral (capsule) 4-AP (10 mg b.i.d. or t.i.d.) over a 4 month interval. The three patients (two male: 1 female) all had incomplete tetraplegia (ASIA levels C and D) with the neurological level of the lesion between C5-C7. Following the administration of 4-AP the patients demonstrated marked and sustained reductions in upper $(n=1)$ or lower extremity $(n=2)$ spasticity. Other clinical benefits of 4-AP were reduced pain $(n=1)$, restored muscle strength $(n=3)$, improved sensation $(n=2)$, voluntary control of bowel function $(n=1)$, and sustained penile tumescence $(n=2)$. The patients exhibited improved hand function $(n=1)$, enhanced mobility in transfers and gait $(n=2)$, with improved energy and endurance. Only trivial side effects (transient lightheadedness) were observed. In one case, the enhanced neurological function allowed the patient to stand with support for the first time post injury (16 years). The time course of therapeutic response to the initial dose matched the pharmacokinetic elimination profile derived from serum and urine analysis. There was no evidence of renal or hepatic toxicity with prolonged use. These results indicate a therapeutic benefit of oral 4-Aminopyridine in the management of various neurological deficits in a select group of SCI patients.
\end{abstract}

Keywords: spinal cord injury; 4-Aminopyridine; spasticity; motor evoked potentials; pharmacokinetics

\section{Introduction}

4-Aminopyridine (4-AP) is a $\mathrm{K}^{+}$channel blocking agent that has been reported to induce transient neurological gain in patients with chronic spinal cord injury (SCI). ${ }^{{ }^{-5}}$ 4-AP blocks fast, voltage-gated (A current) $\mathrm{K}^{+}$channels in demyelinated internodes of central axons. ${ }^{6-8}$ This blockade prolongs the duration of the action current and increases the safety factor for conduction thereby ameliorating conduction deficits due to demyelination. ${ }^{9,10} \mathrm{~K}^{+}$channel blockade also results in increased $\mathrm{Ca}^{2+}$ influx at the dendritic terminals, with attendant increase in transmitter release, in a wide range of neuronal subtypes e.g. GABA-ergic, Cholinergic and adrenergic neurones. $11-15$

The present report describes three patients with SCI who were 'responders' to i.v. 4-AP in the preclinical trials and who were subsequently administered 4-AP orally (10 mg capsule b.i.d. or t.i.d.) over a 4 month period.

Correspondence: PJ Potter
Methods

Patients

The clinical characteristics of the SCI patients are provided in each case report. The patients' neurological deficits were stable and had been quantitatively assessed periodically for at least 12 months prior to this trial. No patient was taking any other medication at the time of entry to the trial. The patients were administered the investigational new drug 4-AP under approval from the Health Protection Branch of the Government of Canada (EDRP\#P60508).

\section{4-Aminopyridine}

Ten mg (10 mg) 4-AP (Regis Chemical Co, Illinois, USA) and lactose was prepared in \#4 gelatin capsules under sterile conditions. This preparation showed a relatively rapid degradation of 4-AP in the capsule within weeks $[\mathrm{Y}=100-2.1(\mathrm{x})$ where $\mathrm{Y}=\%$ 4-AP remaining and $\mathrm{x}$ is weeks since manufacture] ie, a short shelf life, probably due to polymerization (plasticizing interaction betwen 4-AP and gelatin). This necessitated administration of the drug within 20 days of manufacture. 
Protocol

On Day 1 patients received a single $10 \mathrm{mg}$ capsule of 4-AP. Prior to taking the capsule they underwent a physical examination (including ASIA motor and sensory classification and modified Ashworth Scale rating of spasticity), electrophysiological examination including recording of motor evoked potentials (MEPs) following transcranial magnetic stimulation of motor cortex, and assessment of lower limb spasticity. The patients also provided urine and blood samples for illicit drug screen and pharmacokinetic analyses. The electrophysiological and pharmacokinetic assessments were repeated during the $24 \mathrm{~h}$ following drug ingestion.

On Day 4 the patients commenced taking 4-AP b.i.d. They were titrated to t.i.d. over a three day interval, depending on tolerance. Two patients (\#1 and \#3) tolerated t.i.d. while patient \#2 reported wakefulness at night and chose to remain on b.i.d. The patients returned for repeat prescription on a biweekly basis over the course of 4 months and were assessed intermittently on a p.r.n. basis. Each patient maintained a personal record of positive and adverse events. The trial was limited to 4 months for fiscal and administrative reasons.

\section{Pharmacokinetics}

Pharmacokinetic parameters including maximum plasma concentration $\left(\mathrm{C}_{\max }\right) \mathrm{ng} / \mathrm{ml}$; total systemic clearance (CL), L/h: Volume of distribution at steady state (Vss), $\mathrm{L}$; Elimination half life $\left(\mathrm{t}_{1 / 2}\right)$, $\mathrm{h}$ were estimated using a noncompartmental analysis of the time-course of 4-AP in plasma following the initial administration of $10 \mathrm{mg}$ oral 4-AP.

\section{Quantitative assessment of hypertonus}

Lower limb hypertonus was also assessed quantitatively by recording the involuntary mechanical resistance of the foot to passive sinusoidal displacement imposed by a custom designed torque microstepping motor. The foot was displaced through 5 degrees (2.5 degrees each of plantar flexion and dorsiflexion about a neutral axis) at $0.5,1.0,1.5$ and $2.0 \mathrm{~Hz}$. Details of the reliability and sensitivity of this protocol are reported elsewhere. ${ }^{16}$ The total resistive torque $(\tau)$ recorded from a strain gauge, was plotted against angular displacement $(\theta)$, recorded from a potentiometer, to yield a hysteresis loop. Outcome measures from these recordings included the mechanical stiffness $(\Delta \tau / \Delta \theta$ at $0.5 \mathrm{z}$, the velocity-dependent torque contribution measured as the area contained within the hysteresis loop $\left(\int \tau_{\mathrm{p}}-\tau_{\mathrm{d}} \mathrm{d} \theta\right)$ and peak $\tau$ at $\theta=2.5$ degrees dorsiflexion.

\section{Cortical stimulation}

Transcranial magnetic stimulation of the motor cortex was delivered from a Cadwell MES-10 stimulator
(Cadwell Labs, Kennewick, WA, USA) through a focal point stimulating coil electrode with effective radius $4.75 \mathrm{~cm}$. The coil was positioned tangential to the scalp with the rim over $\mathrm{Cz}$ (vertex) of the 10-20 international system for EEG recording. ${ }^{17}$ The orientation of the stimulating coil was maintained constant to ensure the same initial direction of current and the intensity of stimulation was systematically incremented (5\% increments of maximal stimulator output). Stimulation threshold was determined when MEPs were evoked in $\geqslant 50 \%$ of trials. All patients comfortably tolerated stimulation up to $100 \%$ of maximum stimulator output. The methods we used for recording MEPs have been described in detail elsewhere. ${ }^{3,18,19}$

In many instances the amplitude of the MEPs was low and the evoked responses were poorly distinguished from background EMG activity when contractions were employed to facilitate the response. ${ }^{13,18}$ To aid in MEP identification an automated detection algorithm was used which established the criterion for presence of an MEP as a signal with a $1.5 \times$ standard deviation departure from baseline activity $>10 \mathrm{~ms}$ following signal rectification and averaging. The baseline activity was determined in the time window of $2.5-22.5 \mathrm{~ms}$ following cortical stimulation. ${ }^{18}$

\section{Results}

Case 1

Case 1 was a 45 year old $(\mathrm{Ht}=175 \mathrm{~cm} ; \mathrm{Wt}=88.6 \mathrm{~kg})$ man with ASIA Class $\mathrm{C}$ incomplete tetraplegia and Motor Index Score of 49 as a result of a C7 fracture sustained in a motor vehicle accident 16 years before the study. MRI at the time of the study revealed myelomalacia with microcyst formation from C7 to T2. This patient was fully independent at the wheelchair level. He used digital stimulation for bowel management and bladder emptying was accomplished with Credé manoeuvre. In the sixteen years since his injury, he had experienced little improvement in function aside from the occasional ability to actively flex his hip (grade I on the MRC Scale) depending on the current extent of his spasticity. He experienced lumbar pain during sitting in the wheelchair (and when driving) and experienced discomfort with other static positions, including lying in bed, necessitating frequent change in position $(<\min$ when awake). Limitations in daily function were common secondary to spasticity. Spasm resulted in leg movements during the night which would wake him and were uncomfortable to his spouse. During the day, spasms caused instability within the wheelchair due to spontaneous forward body lurches. Similarly, when using hand controls to drive his motor vehicle, spasms would often result in uncontrolled foot contact with the pedals if a physical barrier was not present. His positive response to iv 4-AP (10 mg immediate release) has been reported previously. ${ }^{3}$ 
At the outset of the 4 months of oral administration of 4-AP t.i.d., this man experienced an improvement in motor control of his right leg. He was able to flex his hip (grade 2 on the MRC Scale) and extend his leg at the knee. Motor function was improved to the greatest extent $1-2 \mathrm{~h}$ after each oral dose. His restored leg movement was retained throughout the 4 month trial but was lost after washout.

The improved motor function was associated with reduced spasticity and renewed vigour (no lethargy). He exhibited markedly reduced tone in lower limb musculature, allowing greater passive range of movement and facilitating transfers. He exhibited fewer and less intense spasms thereby enabling better sleep and return to his home-based carpentry work. The reduction in spasticity was sufficient to allow him, for the first time, to pull himself up to a standing position and lock his knees and hips while providing support with his upper limbs.

He experienced appreciable reductions in dysesthetic lumbar pain. Pain in the hip and lower lumbar spine had been treated unsuccessfully over twelve years with a cross section of analgesic modalities and wheelchair positioning. Surgical treatment was being considered to help alleviate the pain. The area of lumbar pain was insensate. After a longstanding inability to tolerate more than one hour of driving in a seated position, this gentleman was now (within first week of 4-AP) able to tolerate three to four hours of driving.

Bladder voiding occurred under voluntary control without the Credé manoeuvre being necessary. He reported a longer holding time (ie, reduced urgency). There was also improved sensation of bowel fullness and a change to minimal and sometimes no rectal stimulation required for defecation. He reported positive quality of life changes associated with his increased confidence and dignity with respect to bowel control.

Sexual function improved with greater duration and strength of penile erection and erections occurring on a psychogenic as well as reflexogenic basis. Prior to drug administration, erections occurred only with direct stimulation. Morning erections increased in frequency with an associated increase in libido.

On termination of the trial this man's spasticity, dysesthetic pain, bladder and bowel control and sexual function regressed to their pre-trial levels over the course of two weeks. This regression was accompanied by marked despondency and lessened capability to do his home-based carpentry.

\section{Case 2}

Case 2 was a 41 year old woman $(\mathrm{Ht}=170.2 \mathrm{~cm}$; $\mathrm{Wt}=71.8 \mathrm{~kg}$ ) who sustained a C5-6 fracture subluxation as a result of a motor vehicle accident 17 months before the study. She had a C6 incomplete ASIA class $\mathrm{D}$ tetraparesis and a Motor Index score of 70. Clinical examination revealed diminished sensation below C6 and motor loss below $\mathrm{C} 8$, with the most extensive paresis present in the intrinsic muscles of the hand and proximally in the lower left extremity. Electrophysiological investigations revealed a well-defined tibial nerve cortical SEP of moderate amplitude. Low amplitude MEPs were recorded bilaterally from TA and LG only with reinforcement from target muscle contraction, and sparse, low amplitude EMG interference patterns were recorded bilaterally on voluntary effort.

After the initial dose of $10 \mathrm{mg}$ oral 4-AP, this woman demonstrated clear enhancement of MEP's in lower limb muscles following cortical stimulation. There were reduced stimulation thresholds and increased amplitude responses in each of the muscles monitored (left lateral gastrocnemius, left extensor digitorum brevis muscle and left tibialis anterior) in the more paretic left leg. The latency of evoked response was also reduced in all of these muscles. These changes were evident from recordings made with the muscle at rest, or when contracted. Figure 1 illustrates the increase in MEP amplitude recorded from the extensor digitorum muscle before and after administration of 4-AP.

Over the course of the 4 month trial, this woman exhibited marked improvements in gait, manifest by increased stability, foot clearance, coordination and endurance. This was associated with increased sensation, strength, and reduced tone in her legs bilaterally. Her upper extremeties exhibited less tone, allowing greater manual dexterity (finger extension, abduction and adduction), increased touch sensation and reduced fatigue. This allowed increased performance in her part-time labour on the assembly line. She reported improved mood. The type of changes were similar to those reported following IV 4-AP single dose administration $^{3}$ but the extent was greater and was sustained throughout the 4 months.

After the completion of the trial this woman's abnormal tone reverted to its pre-drug state over the course of one week.

Case 3

Case 3 was a 40 year old man $(\mathrm{Ht}=182.9 \mathrm{~cm}$; $\mathrm{Wt}=79.6 \mathrm{~kg}$ ) who sustained $\mathrm{C} 4-5$ tetraplegia in a diving accident three years prior to the study. He presented with spastic gait, assisted by canes, and was capable of walking only short distanes. He required a wheelchair to travel longer distances. He reported frequent spasms and clonus in his legs. He had recently been treated for autonomic dysreflexia. He was ASIA class D with a Motor Index Score of 77 and Functional Independence Measure (FIM) score of 111 . He exhibited a marked increase in tone at his ankle joint. The values for his resistance to passive dorsiflexion and plantarflexion exceeded normal control values $(Z>1.64)$.

This man exhibited marked reduction in tone in his lower limbs within $2 \mathrm{~h}$ of the first dose of oral 4-AP. Quantitative assessment of his ankle joint hypertonus 


\section{Effects of 4-Aminopyridine on Motor Evoked Potentials}
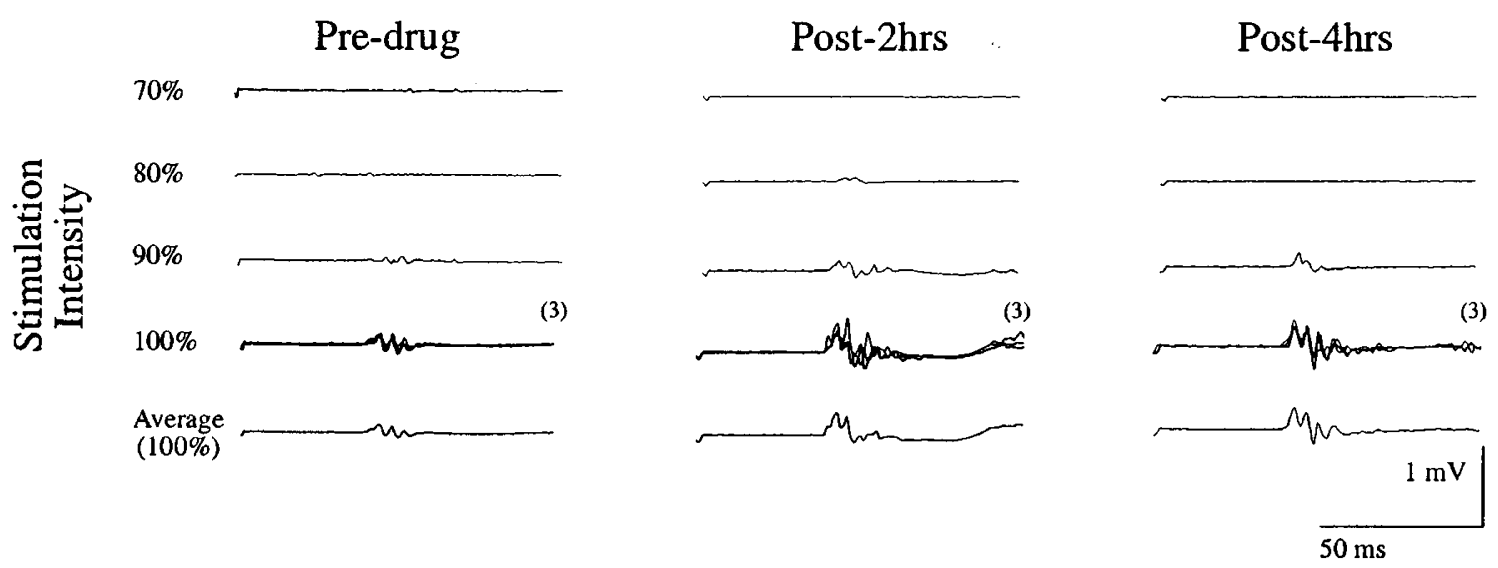

Figure 1 Motor evoked potentials (MEPs) recorded from the relaxed extensor digitorum muscle prior to and following administration of $10 \mathrm{mg}$ oral 4-Aminopyridine (4-AP) in a 41 year old female C5-6 incomplete tetraplegic patient (Case \#2). The amplitude of the MEP was markedly increased at both $2 \mathrm{~h}$ and $4 \mathrm{~h}$ after drug administration. The stimulation threshold (expressed as \% maximum stimulator output) and MEP latency were reduced after receiving 4-AP. Numbers in parenthesis indicate number of traces superimposed and contained in the ensemble average (bottom trace)

revealed appreciably less resistance to passive angular displacement at all frequences tested $(0.5-2.0 \mathrm{~Hz})$. Figure 2 illustates this reduction in tone, shown as reduced slope, reduced peak resistive torque $(\tau)$, and reduced area contained within the hysteresis loop $\left(\int \tau_{\mathrm{d}}-\right.$ $\left.\tau_{\mathrm{p}} \theta\right)$.

Case \#3 exhibited increased amplitude MEPs in all muscles examined at $2 \mathrm{~h}$ and $4 \mathrm{~h}$ following $10 \mathrm{mg}$ 4AP. The stimulation threshold for evoking an MEP was reduced as was the latency of the evoked response. Responses obtained from the left extensor digitorum brevis (EDB) are illustrated in Figure 3. This patient also exhibited reduced amplitude F-waves in the left EDB indicative of reduced motoneurone excitability. This observation suggests that 4-AP induced an increase in descending excitatory inputs to the motoneurone pool, sufficient to overcome the reduction in motoneurone excitability and to yield the increased amplitude MEP. The reduction in F-wave amplitude (and motoneurone excitability) is consistent with the quantitative evidence of reduced hypertonus and the clinical observations of reduced spasticity.

The reduced tone and increased coordination parallelled functional improvements in gait, stair climbing, transfers, confidence and endurance. He reported increased muscle strength of hand muscles and increased grip strength as a result of reduced tone. This was evident as greater finger extension without cocontraction of flexor muscles, and increased finger abduction and adduction and pinch strength. He also noted increased sitting tolerance as a result of reduced tone. This man reported increased frequency and duration of penile erections. A non-problematic side effect of this drug was irritability and wakefulness at night which was eliminated by reduction from t.i.d. to

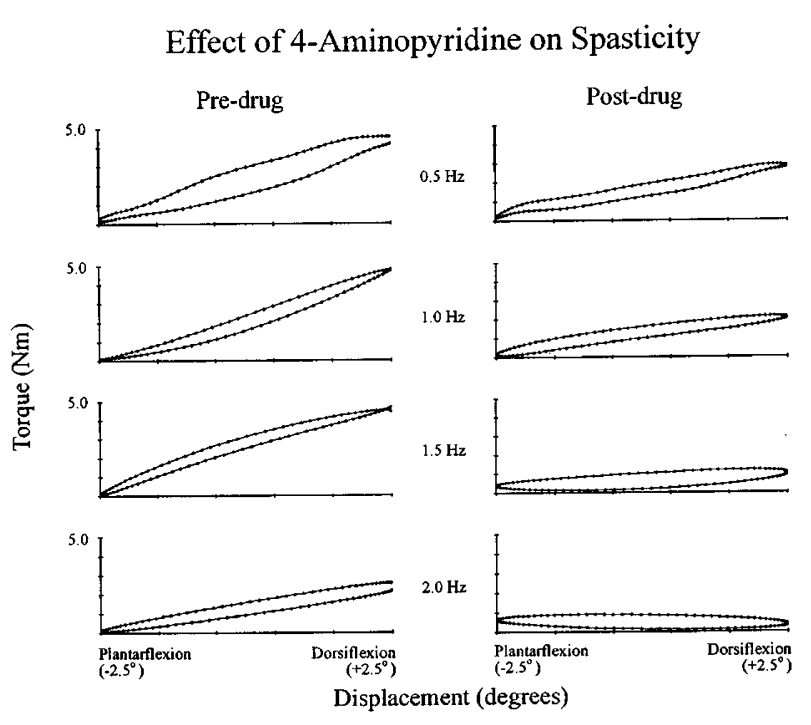

Figure 2 Changes in ankle joint hypertonus following administration of a single dose $(10 \mathrm{mg})$ of 4-AP in Case \#2. Each trace records the average hysteresis loop formed by plotting the total resistive torque about the ankle joint as a function of the angular displacement over five cycles. The foot was passively displaced at $0.5 \mathrm{~Hz}-2.0 \mathrm{~Hz}$ through $\pm 2.5 \mathrm{deg}$. There was an appreciable reduction in the slope $(\Delta \tau / \Delta \theta)$ indicating reduced stiffness at each frequency

b.i.d. administration during the dose titration phase. He reported a sense of 'well-being' while on 4-AP, b.i.d.

On completion of the trial this man's neurological status reverted to its pre-trial status over the course of two weeks. 


\section{Effects of 4-Aminopyridine on Motor Evoked Potentials}
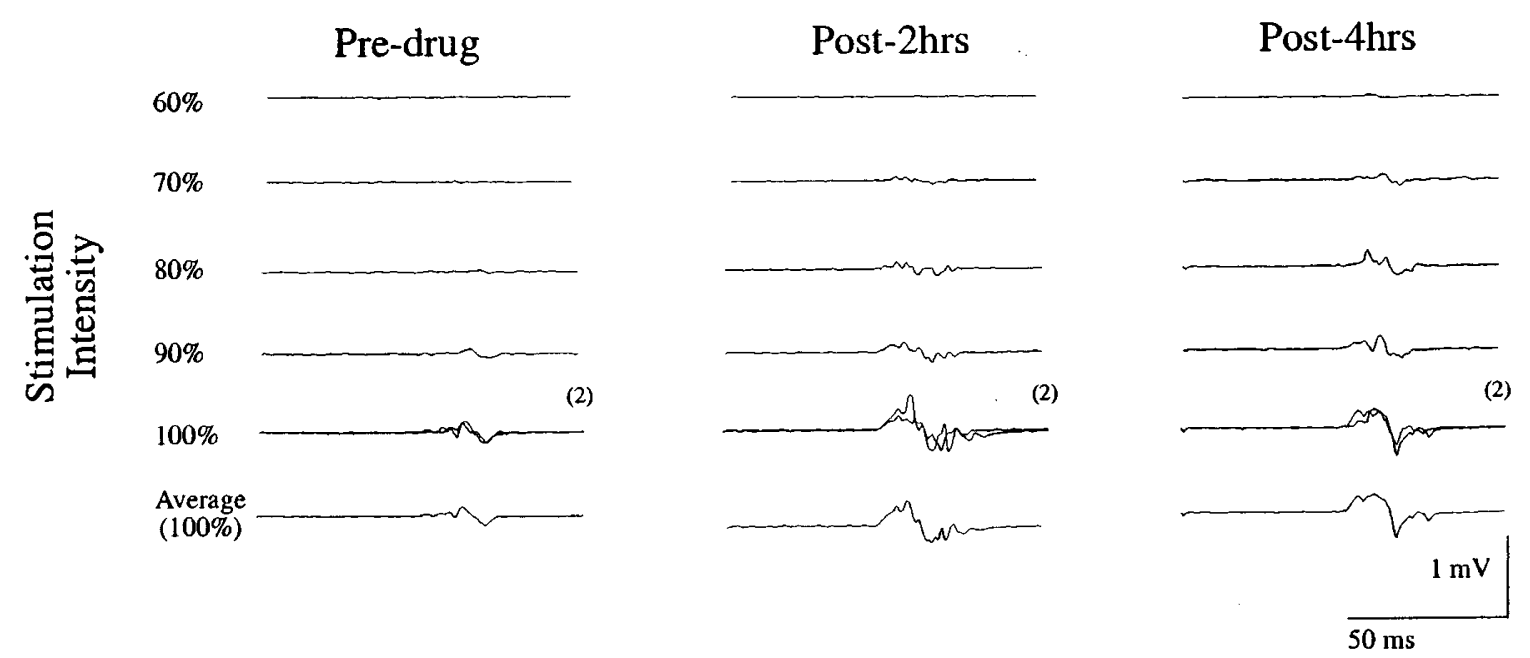

Figure 3 Motor evoked potentials (MEPs) recorded from the relaxed extensor digitorum muscle prior to and following $10 \mathrm{mg}$ oral 4-Aminopyridine (4-AP) in a 40 year old male with C5-6 incomplete tetraplegia (Case \#3). The amplitude of the MEP was markedly increased at both $2 \mathrm{~h}$ and $4 \mathrm{~h}$ after drug administration. The stimulation threshold (expressed as \% maximum stimulator output) and MEP latency were reduced after receiving 4-AP. Numbers in parentheses indicate number of traces superimposed and contained in the ensemble average (bottom trace)

\section{Pharmacokinetic analysis}

The time-course of plasma concentrations of 4aminopyridine (4-AP) was followed for up to $36 \mathrm{~h}$ in two patients and for $24 \mathrm{~h}$ in the third. The 4-AP concentration-time profiles are graphically depicted in Figure 4. The pharmacokinetic parameters estimated from a noncompartmental kinetic analysis are displayed in Table 1. 4-AP plasma concentrations reproducibly oscillated over time in each of the three subjects. The oscillations in measured drug levels were most easily discerned during the sampling rich interval that extended for $6 \mathrm{~h}$ post 4-AP administration. Peak plasma levels of 4-AP $\left(\mathrm{C}_{\max }\right)$ were observed less than two hours after drug administration (Table 1). Total systemic clearance (CL) and volume of distribution at steady-state (Vss), estimated from an area-moment analysis, ranged from $1.76 \mathrm{~L} / \mathrm{h}$ to $2.41 \mathrm{~L} / \mathrm{h}$ and $139.8 \mathrm{~L}$ to $306.5 \mathrm{~L}$, respectively. These parameters agreed, within an order of magnitude, with previously published values estimated from a multiexponential kinetic model. ${ }^{20} \mathrm{~A}$ noncompartmental kinetic analysis and extended measurements of 4-AP in plasma for up to $36 \mathrm{~h}$, as carried out in this study, supported a more accurate estimation of the rate of disappearance of 4AP from the systemic circulation than has previously been reported in SCI. Plasma levels experimentally measured in our patients, when contrasted to estimates of 4-AP concentrations extrapolated from multiexponential (multicompartmental) kinetic models, decayed with a terminal elimination half-life $\left(t_{1 / 2}\right)$ in excess of $16 \mathrm{~h}$ in contrast to a currently accepted literature estimate of approximately $3.6 \mathrm{~h}^{20}$ and are thus more supportive of, and correlate better with, clinical observations of prolonged 4-AP effects following a single dose. ${ }^{4}$

\section{Time course of effects}

The time course of effects of 4-AP varied for the different indices of therapeutic benefit. Sensory, analgesic and antispastic effects tended to appear $1-$ $2 \mathrm{~h}$ after the first dose consistent with the peak serum levels. Other properties such as the improved bowel and bladder function only became evident at times when the bowel routines were implemented.

\section{Discussion}

4-Aminopyridine has a long history of clinical use; first to antagonize neuromuscular blockade from various anaesthetics, ${ }^{22-24,66}$ then as a therapeutic agent to enhance neural or neuromuscular transmission in patients with Alzheimers disease, ${ }^{25}$ botulinum toxicity, ${ }^{26}$ myaesthenia gravis ${ }^{27,28}$ or Eaton-Lambert Syndrome ${ }^{21,29}$ and more recently as a means of overcoming central conduction deficits due to demyelination in patients with multiple sclerosis. ${ }^{30-40}$ Its application in patients with spinal cord injury is even more recent and is still in the investigative stages as the drug's safety and efficacy have yet to be definitively established in the SCI population by randomized placebo-controlled, double-blind clinical trials. It is against this background that the present open label compassionate use trial of 4-AP was undertaken.

The clinical outcomes witnessed in the present 4 month trial are consistent with previously documented 

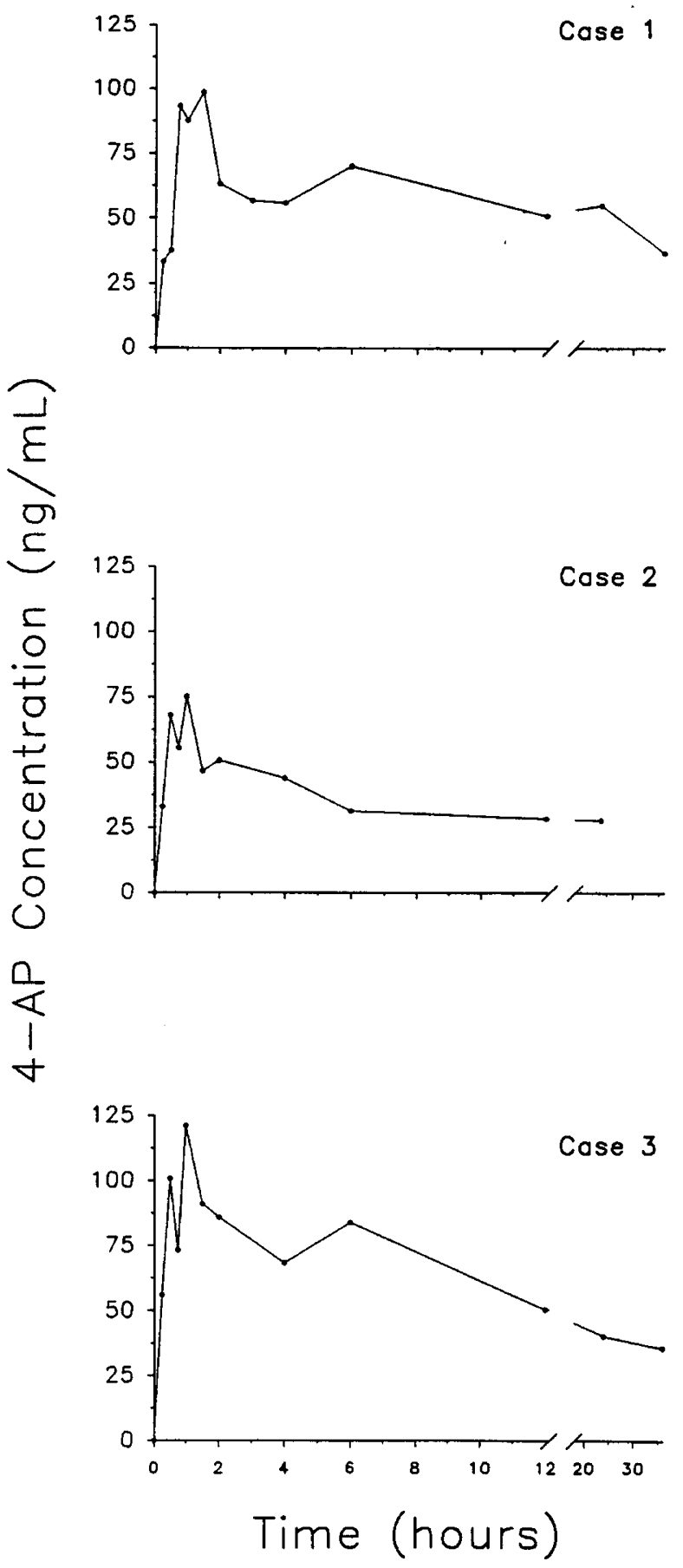

Figure 4 4-AP plasma concentration time-course for the three SCI cases

transient neurological gains following single doses of 4-Aminopyridine. ${ }^{1-4}$ The objective documentation of changes in limb hypertonia (Figure 2) and central motor conduction (Figures 1 and 3 ) following the initial dose of 4-AP adds further support to the accumulating clinical evidence of neurological benefit. Similarly, the pharmacokinetic profile reported here
Table 1 4-Aminopyridine pharmacokinetic parameters

\begin{tabular}{lccccc}
\hline Case & $T_{\max }(h)$ & $C_{\max }(n g / m l)$ & $C L(L / h)$ & $V_{s s} /(L)$ & $t_{1 / 2}(h r)$ \\
\hline 1 & 1.5 & 98.82 & 5.21 & 139.84 & 18.73 \\
2 & 1.0 & 75.08 & 12.61 & 306.48 & 16.90 \\
3 & 1.0 & 121.27 & 5.34 & 153.08 & 19.08 \\
\hline
\end{tabular}

$\tau_{1 / 2} \equiv$ half-life of the drug in plasma. $\mathrm{T}_{\max } \equiv$ time to maximum plasma concentration. $\mathrm{C}_{\max } \equiv$ maximum plasma concentration. $\mathrm{CL} \equiv$ total systemic clearance. Vss $\equiv$ volume of distribution at steady-state

(Figure 4) for the initial dose confirms previous reports. $^{4}$ More importantly, the effects of multiple dosing revealed a cumulative therapeutic effect over the first few days, followed by some sustained benefit over four months, consistent with the demonstration of a longer terminal elimination half-life than previously described (Table 2). The oscillation in measured plasma levels of 4-aminopyridine observed by us and others ${ }^{4}$ can reasonably be attributed to enterosystemic recirculation, and 4-AP is thought to undergo little, if any, biotransformation. Hence, enterosystemic recirculation of 4-AP, perhaps exaggerated in humans with SCI, can be postulated as the cause of the longer elimination half-life that we observed and can be implicated as the putative mechanism mediating the prolonged elimination phase and subsequent cumulative or sustained clinical effects. ${ }^{41}$

The functional benefits to the three patients were diverse. One of the patients (Case \#1) returned to work in his carpentry shop. He had previously been restricted by spasticity and pain. A second patient (Case \#2) reported better manual dexterity as a consequence of reduced upper limb spasticity enabling her to function more effectively in her assembly line employment. These outcomes, together with improved bowel and bladder function, reduced pain and improved motor function in gait and a nonspecific but consistently reported 'renewed vigour', collectively added appreciably to the dignity and quality of life of these individuals.

How can a single pharmacological agent, with relatively simple molecular structure, introduce change in so many diverse symptoms and physiological subsystems? There are two principal means by which the $\mathrm{K}^{+}$channel blocking action of 4-AP may effect change. The first is by overcoming central conduction deficits due to demyelination; the second, by increasing $\mathrm{Ca}^{2+}$ influx at presynaptic sites to increase neurotransmitter release and enhance neural or neuromuscular transmission. ${ }^{13,22,42}$ The latter property has been previously shown to be effective in a wide variety of neural subsystems and species. It may account for analgesia ${ }^{43}$ unmasking ineffective synapses ${ }^{44}$ increased discharge in sensory neurones ${ }^{45,46}$ and increased receptive fields in dorsal horn, gracile and cuneate neurones, ${ }^{44,47}$ enhanced presynaptic inhibition (primary afferent depolariza- 
Table 2 Physiological bases of clinical outcomes

\begin{tabular}{llc}
\hline Clinical Outcome & Potential Physiological Mechanism & Reference \\
\hline Increased Motor Function & restored conduction in demyelinated axons & 8,10 \\
& increased neuromuscular transmission [Ca ${ }^{+}$influx increases] & 15,62 \\
& increased contractility: excitation-contraction coupling & 11 \\
& increase in L-Dopa induced fictive locomotion & 61 \\
Increased Sensation & altered receptive field of cuneate and gracile neurones & 45 \\
& increased spontaneous sensory discharge & 45 \\
& enhanced receptor discharge & $44,46,47,63$ \\
Reduced Pain & increased receptive field of dorsal horn neurones & 44 \\
& morphine-like analgesia & 43 \\
Reduced Spasticity & activation of type 2 receptors via serotonergic system & 64 \\
Penile Tumescence & GABA-ergic properties & $48,49,50,51$ \\
Bowel and Bladder Control Improved & enhanced primary afferent depolarization & 15 \\
& parasympathomimetic properties & 11 \\
\hline
\end{tabular}

tion $)^{48-51}$ and sympathomimetic or parasympathomimetic properties. ${ }^{11,15,52}$ Table 2 summarizes the putative physiological bases for the clinical outcomes observed in the present study.

In summary, the 4 month compassionate use trial of $10 \mathrm{mg}$ b.i.d. oral 4-Aminopyridine (immediate release) in three SCI patients has confirmed and extended previous reports of potential therapeutic benefit of this compound in both animal models of $\mathrm{SCI}^{53-55}$ and man. $^{2-4}$ Multidosing led to appreciable functional gains for these patients in diverse areas including manual dexerity, ambulation, transfers, bowel and bladder function, and was associated with a generalized sense of wellbeing. Side effects were negligible in this trial.

Previous reports of the long-term administration of oral 4-AP in Multiple Sclerosis patients have cautioned of the need for careful medical supervision during 4-AP therapy. Polman et $a l^{35}$ reported two major side-effects of (a) tonic clonic epileptic seizures $(n=2)$ and (b) presumed 4-AP induced hepatitis $(n=1)$ in a group of 23 patients receiving oral 4-AP for $6-32$ months. This, and other reports of overdosing with 4AP in healthy adults and MS patients in uncontrolled circumstance ${ }^{56-58}$ have prompted the suggestion that serum concentration control may be a useful therapeutic strategy. 31

Interpretation of the overall efficacy of oral 4-AP, as documented in the present compassionate use application, is obviously constrained by (a) the pre-selection of responsive patients and (b) the absence of any placebo control. Acknowledging those caveats, it is noteworthy that, prior to receiving 4-AP, the patients' neurological status had been well established as stable. All three patients were beyond the time frame since injury ( $>2$ years) when appreciable spontaneous functional improvement would be expected. Indeed, one patient had a 16 year 'historical control'. Definitive evidence of a generalized therapeutic benefit of 4-AP in SCI clearly must await multicenter placebo-controlled clinical trials. Such studies are now underway.

\section{References}

1 Hayes $\mathrm{KC}$ et al. Effects of intravenous 4-aminopyridine on neurological function in chronic spinal cord injured patients: Preliminary observations. Proc IBRO World Conf Neurosci 1991b; 345:(Abstr) P53.27.

2 Hayes KC. et al. Preclinical trial of 4-aminopyridine in patients with chronic spinal cord injury. Paraplegia 1993; 31: 216-224.

3 Hayes KC et al. 4-aminopyridine-sensitive neurologic deficits in patients with spinal cord injury. J Neurotrauma 1994; 11: 433 446.

4 Hansebout RR, Blight AR, Fawcett S, Reddy K. 4-aminopyridine in chronic spinal cord injury: a controlled, double-blind, crossover study in eight patients. J Neurotrauma 1993; 10: $1-8$.

5 Waxman SG. Aminopyridines and the treatment of spinal cord injury. J Neurotrauma 1993; 10: 19-24.

6 Bostock H, Sears TA, Sherratt RM. The effects of 4aminopyridine and Tetraethylammonium ions on normal and demyelinated mammalian nerve fibres. J Physiol (Lond) 1981; 313: $301-315$.

7 Bowe CM, Kocsis JD, Targ EF, Waxman SG. Physiological effects of 4-aminopyridine on demyelinated mammalian motor and sensory fibers. Ann Neurol 1987; 22: $264-268$.

8 Sherratt RM, Bostock H, Sear TA. Effects of 4-aminopyridine on normal and demyelinated mammalian nerve fibers. Nature 1980; 283: $570-572$.

9 Blight AR. Effect of 4-aminopyridine on axonal conductionblock in chronic spinal cord injury. Brain Res Bull 1989; 22: 47 52.

10 Targ EF, Kocsis JD. 4-aminopyridine leads to restoration of conduction in demyelinated rat sciatic nerve. Brain Res 1985; 328: $358-361$. 
11 Bowman WC, Savage AO. Pharmacological actions of aminopyridine and related compounds. Rev Pure Appl Pharmacol 1981; 2: $317-371$.

12 Glover WE. The Aminopyridines. Gen Pharmacol 1982; 13: 259 285.

13 Hayes KC. 4-aminopyridine and spinal cord injury: a review. Restor Neurol \& Neurosci 1994; 6: 259-270.

14 Soni N, Kam P. 4-aminopyridine - A review. Anaesth Intens Care 1982; 10: $120-126$.

15 Thesleff S. Aminopyridine and synaptic transmission. Neurosciences 1980; 5: 1413-1419.

16 Gardner DM, Hayes KC, Delaney GA, Potter PJ (1996) Biomechanics of ankle joint hypertonus in spinal cord injured patients: An analytic approach to quantifying spasticity. Proceedings Australasian Biomechanics Conference, Univ. of Sydney, Australia, February $1-2$.

17 Jasper HH. The ten-twenty electode system of the International Federation. Electroencephalogr Clin Neurophysiol 1958; 10: 371 375.

18 Wolfe DL, Hayes KC, Potter PJ, Delaney GA. Conditioning lower limb H-reflexes by transcranial magnetic stimulation of motor cortex reveals preserved innervation in SCI patients. $J$ Neurotrauma 1996; 13: $281-291$.

19 Qiao J et al. Effects of 4-aminopyridine on motor evoked potentials in patients with spinal cord injury. J Neurotrauma 1997; 14: $125-149$.

20 Uges DRA et al. 4-aminopyridine kinetics. Clin Pharmacol Ther 1982; 31: $587-593$.

21 Agoston S, Van Weerden T, Westra P, Brockert A. Effects of 4aminopyridine in Eaton Lambert Syndrome. Br J Anaesth 1978; 50: $383-385$

22 Booij IHDJ, Miller RD, Crul JF. Neostigmine and 4-aminopyridine antagonism of lincomycin-pancuronium neuromuscular blockade in man. Anaesth Anal 1978; (Clev) 57: 316-321.

23 Paskov DS, Agoston S, Bowman WC. 4-aminopyridine hydrochloride (Pymadin) (1986) In: New Neuromuscular Blocking Agents - Basic and Applied Aspects, DA Charkavich (Ed), Handbook of Experimental Pharmacology, Vol 870, SpringerVerlag, pp. $679-717$

24 Sia RI et al. Effects of the analeptic drug, 4-aminopyridine upon postoperative respiratory depression in patients, A preliminary study. Acta Anaesth Belg 1979; 30(Suppl): 195-199.

25 Wesseling H, Agoston S. Effects of 4-aminopyridine in elderly patients with Alzheimer's Disease. The New Engl J Med 1984; 310: $988-989$.

26 Ball AP et al. Human botulism caused by clostridium botulinum type e - The Birmingham Outbreak. Quart J Med 1979; 48: $473-$ 491.

27 Murray NMF, Newsom-Davis J. Treatment with oral 4aminopyridine in disorders of neuromuscular transmission. Neurology 1981; 31: 265-271.

28 Lundh H, Nilsson O, Rosen I. Effects of 4-aminopyridine in myasthenia gravis. J Neurol Neurosurg Psychiatry 1979; 42: 171 175

29 Lundh H, Nilsson O, Rosen I. 4-aminopyridine - A new drug tested in the treatment of Eaton-Lambert syndrome. J Neurol Neurosurg Psychiatry 1977; 40: 1109-1112.

30 Bever CT Jr. The current status of studies of animopyridines in patients with multiple sclerosis. Annals of Neurology 1994; 36(Suppl): S118-121.

31 Bever CT Jr et al. The effects of 4-aminopyridine in multiple sclerosis patients: Results of a randomized, placebo-controlled, double-blind, concentration-controlled, crossover trial. Neurology 1994; 44: $1054-1059$.

32 Davis FA, Stefoski D, Rush J. Orally administered 4aminopyridine improves clinical signs in multiple sclerosis. Ann Neurol 1990; 27: 186-192.

33 Davis FA, Stefoski D, Quandt FN. Mechanism of action of 4 aminopyridine in the symptomatic treatment of multiple sclerosis. Annals of Neurology 1995; 37: 684.

34 Jones RE et al. Effects of 4-aminopyridine in patients with multiple sclerosis. J Neurol Sci 1983; 60: $353-362$.
35 Polman CH, Bertelsmann FW, van Loenen AC, Koetsier JC. 4 aminopyridine in the treatment of patients with multiple sclerosis: long-term efficacy and safety. Arch Neurol 1994; 51: $292-296$.

36 Polman $\mathrm{CH}$ et al. 4-aminopyridine is superior to 3,4-Diaminopyridine in the treatment of patients with multiple sclerosis. Arch Neurol 1994; 51: 1136-1139.

37 Smits RC et al. The effects of 4-aminopyridine in cognitive function in patients with multiple sclerosis: A pilot study. Neurology 1994; 44: 1701 - 1705

38 Stefoski DF, David FA, Faut M, Schauf CL. 4-aminopyridine improves clinical signs in multiple sclerosis. Ann Neurol 1987; 21: $71-77$.

39 Stefoski D et al. 4-aminopyridine improves clinical signs in multiple sclerosis. Ann Neurol 1991; 21: $71-77$.

40 van Diemen HA et al. The effect of 4-aminopyridine on the clinical signs in multiple sclerosis: a randomized, placebocontrolled, double-blind, crossover study. Ann Neurol 1992; 32. $123-130$.

41 Segal JL, Brunneman SR, Eltorai IM, Vulpe M. Decreased systemic clearance of lorazepam in humans with spinal cord injury. J Clin Pharmacol 1991; 31: 651-656.

42 Felts PA, Smith KJ. The use of potassium channel blocking agents in the therapy of demyelinating disease. Annals of Neurology 1994; 36: 454

43 Fastier FN, McDowall MA. Analgesic activity of 4-methyl-2amino-pyridine and of some related compounds. Australian $J$ Biological Sci 1958; 36: 491 - 498

44 Saade N, Jabbur SJ, Wall PD. Effects of 4-aminopyridine, GABA and bicuculline on cutaneous receptive fields of cat dorsal horn neurons. Brain Research 1985; 344: 356 - 359.

45 Kocsis JD, Bowe CM, Waxman SG. Different effects of 4 aminopyridine on sensory and motor fibers: Pathogenesis of paresthesias. Neurology 1986; 36: $117-120$.

46 Kirchoff C, Leah JD, Jung S, Reeh PW. Excitation of cutaneous sensory nerve endings in the rat by 4-aminopyridine and tetraethylammonium. J Neurophysiol 1992; 67: 125 - 131.

47 Saade NE et al. Cutaneous receptive field alterations induced by 4-aminopyridine. Brain Research 1982; 232: $177-180$.

48 Kita T, Kita H, Kitai ST. Effects of 4-aminopyridine (4-AP) on rat neostriatal neurons in an in vitro slice preparation. Brain Research 1985; 361: $10-18$.

49 Jankowska E, Lundberg A, Rudomin P, Sykova E. Effects of 4aminopyridine on synaptic transmission in the cat spinal cord. Brain Research 1982; 240: $117-129$.

50 Lemeignan M. Analysis of the action of 4-aminopyridine on the cat lumbar spinal cord I. Modification of the afferent volley, the monosynaptic discharge, amplitude and the polysynaptic evoked responses. Neuropharmacology 1972; 11: $551-558$.

51 Lemeignan M. Analysis of the effect of 4-aminopyridine on the lumbar spinal cord of the cat - II. Modification of certain spinal inhibitory phenomena post-tetanic potentiation and dorsal root potential. Neuropharmacology 1973; 12: 641-651.

52 Anden NE, Leander S. Effects of 4-aminopyridine on the turnover of monoamines in the central nervous system of the rat. J Neurol Trans 1979; 44: 1 - 12 .

53 Blight AR, Gruner JA. Augmentation by 4-aminopyridine of vestibulospinal free fall responses in chronic spinal-injured cats. J Neurol Sci 1987; 87: 145-159.

54 Blight AR, Toombs JP, Bauer MS, Widmer WR. The effects of 4aminopyridine on neurological deficits in chronic cases of traumatic spinal cord injury in dogs: A Phase I clinical trial. $J$ Neurotrauma 1991; 8: $103-119$.

55 Pratt K, Toombs JP, Widmer WR, Borgens RB. Plasma and cerebrospinal fluid concentrations of 4-aminopyridine following intravenous injection and metered intrathecal delivery in canines. J Neurotrauma 1995; 12: 23-39.

56 Smeets JW, Kunst MW. Severe poisoning by 4-aminopyridine in a body builder. Ned Tijdschr Geneeskd 1995; 139: 2667-2669.

57 Spyker DA, Lynch S,Shabanowitz J, Sinn JA. Poisoning with 4aminopyridine: Report of three cases. Clin Toxicol 1980; 16: 487-497. 
58 Stork CM, Hoffman RS. Characterization of 4-aminopyridine in overdose. J Toxicology - Clinical Toxicology 1994; 32: 584-587.

59 Kim YI, Goldner MM, Sanders DB. Facilitatory effects of 4aminopyridine on normal neuromuscular transmission. Muscle \& Nerve 1980; 3: $105-111$.

60 Molgo J, Lundh H, Thesleff S. Potency of 3,4 diaminopyridine and 4-aminopyridine on mammalian neuromuscular transmissions and the effect of pH changes. Eur J Pharmacol 1980; 61: 25.

61 Zangger $\mathrm{P}$. The effect of 4-aminopyridine on the spinal locomotor rhythm induced by L-Dopa. Brain Research 1981; 215: $211-223$.

62 Molgo J, Lemeignan M, Lechat P. Effects of 4-aminopyridine at the frog neuromuscular junction. J Pharmacol Exp Ther 1977; 203: $653-663$.
63 Saade NE, Chanelet J, Lonchampt P. Action facilitatrice de microinjection de 4-aminopyridine sur les activities medullaries reflexes de la Grenouille. CR Soc Biol 1971; 165: 2069 - 2078.

64 Avoli M, Perreault P, Olivier A, Villemure J-G. 4-aminopyridine induces a long-lasting depolarizing GABA-ergic potential in human neocortical and hippocampal neurons maintained in vitro. Neuroscience Letters 1988; 94: 217-332.

65 Jankowska E, Lundberg A, Rudomin P, Sykova E. Effects of 4aminopyridine on transmission in excitatory and inhibitory synapses in the spinal cord. Brain Research 1977; 136: 387-388.

66 Agostin $\mathrm{S}$ et al. Antagonism of Ketamine-Diazepam anaesthesia by 4-aminopyridine in human volunteers. Br J Anaesth 1980; 52: $367-370$. 